\title{
Clinicopathological Analysis of Contrast-Enhanced Ultrasonography Using Perflubutane in Pancreatic Adenocarcinoma
}

\author{
GEN AKASU, RYUICHI KAWAHARA, MAKIKO YASUMOTO*, TAKENORI SAKAI, \\ YUICHI GOTO, TOSHIHIRO SATO, KENJIRO FUKUYO, KOJI OKUDA, \\ HISAFUMI KINOSHITA AND HIROYUKI TANAKA \\ Department of Surgery, and $*$ Pathology Kurume University School of Medicine, \\ Kurume 830-0011, Japan
}

Received 2 July 2012, accepted 10 December 2012

Edited by MICHIO SATA

\begin{abstract}
Summary: The contrast harmonic imaging technique allows visualization of micro bubbles and has facilitated the detection of blood flow on contrast-enhanced ultrasonography (CE-US). In hypovascular tumors such as pancreatic cancer a hypoxic nutrition-deficient environment increases tumor malignancy. In this study, we investigated the relation between CE-US findings, intratumoral microvessel density (MVD), and pathological analysis in pancreatic cancer, and we also investigated the clinicopathological significance of CE-US.

The subjects were 16 pancreatic cancer patients who underwent CE-US before surgery. A time-signal intensity curve (TIC) was prepared based on the region of interest (ROI) in the tumor on CE-US, and the signal intensity (SI) was defined as an increase from the value before contrast imaging to the maximum value. Regarding MVD, histological sections were stained with anti-CD34 and $\alpha$-smooth muscle actin ( $\alpha$-SMA) antibodies, and double stained micro-blood vessels were counted. The correlation between SI and MVD was investigated. In addition, disease-free survival (DFS) was compared between the hypo ( $\leq$ mean SI) and hyper (>mean SI) SI groups.

$\mathrm{SI}$ in cancerous lesions was $54.6 \pm 42.9 \mathrm{~dB}(\operatorname{mean} \pm \mathrm{SD})$, and MVD in cancerous lesions was $12.5 \pm 5.02$ $($ mean $\pm \mathrm{SD})$. A positive correlation was noted between the SI and MVD $\left(\mathrm{r}^{2}=0.408, \mathrm{p}=0.008\right)$. The median DFS were 212 and 606 days in the hypo and hyper SI groups, respectively, showing a significantly shorter DFS in the hypo SI group $(\mathrm{P}=0.003)$. No patient died of the primary disease during the follow-up period in the hyper SI group, and a maximum 47-month follow-up was possible.

A positive correlation was noted between SI and MVD, indicating that MVD of pancreatic cancer could be evaluated using CE-US. We suggested that CE-US is a useful predictor of patient prognosis after pancreatic cancer surgery.
\end{abstract}

Key words pancreatic adenocarcinoma, contrast-enhanced ultrasonography, time-signal intensity curve, microvessel density, disease-free survival

\section{INTRODUCTION}

Sonazoid ${ }^{\circledR}$ (Daiichi-Sankyo, Tokyo, Japan) is an intravenous contrast medium for ultrasonography (US) comprised of that can easily pass through capillary blood vessels. These micro bubbles are not destroyed by low-acoustic pressure harmonic imaging, therefore allowing real-time visualization of blood flow and continuous observation of circulatory dynamics in large through capillary blood vessels [1]. Contrast-enhanced ultrasonography (CE-US) can be performed in an outpatient consultation room or at the bedside in wards,

Address correspondence to: GEN AKASU, Department of Surgery, Kurume University School of Medicine. E-mail: akasugen@med.kurume-u.ac.jp

Abbreviations: CE-US, contrast-enhanced ultrasonography; MVD, microvessel density; SI, signal intensity; TIC, time-signal intensity curve; ROI, region of interest; $\alpha$-SMA, $\alpha$-smooth muscle actin; DFS, disease-free survival; US, ultrasonography; Ph, pancreas head; Pb, pancreas body; PD, pancreatoduodenectomy; PPPD, pylorus-preserving PD; SSPPD, subtotal stomach-preserving; DP, distal pancreatectomy; OS, overall survival. 
and the incidence of adverse effects of Sonazoid ${ }^{\circledR}$ is $0.5 \%$, showing a high-level of safety [2]. Simplicity and minimal risk of allergic reactions to contrast medium are also characteristics of CE-US using Sonazoid $\AA_{\text {. }}$

Since pancreatic cancer appears as a poorly enhanced mass on contrast CT and MRI [3], micro blood vessels in pancreatic cancer have been pathologically evaluated by measuring the microvessel density (MVD) in excised specimens. CE-US has been shown to be able to accurately evaluate vascularization of solid pancreatic lesions [4,5]. Although several reports have suggested the usefulness of CE-US using Levovist ${ }^{\circledR}$ (Shering, Tokyo, Japan) in pancreatic tumors in clinical practice $[6,7,8]$, it has not become common because of its complicated CE-US method and inefficient visualization. CE-US using Sonazoid ${ }^{\circledR}$, however, allows blood flow in pancreatic cancer to be detected simply and efficiently.

Since Weidner et al. initially measured MVD using anti-factor VIII antibody for the staining of micro blood vessels in 1991 [9], many studies on the association between the prognosis of hyper vascular tumors and MVD have been performed. Assessing tumor vascularity is clinically important because of acknowledged tumor characterization. Many studies are targeting MVD as a prognostic marker of cancer. Pancreatic cancer is an aggressive and devastating disease. In Japan, the prognosis after pancreatectomy even in stage I pancreatic cancer is poorer than that of other cancers, with a 5-year-survival rate of $52.5 \%$ (1991-2000) [10]. Therefore, a more accurate preoperative evaluation of MVD in pancreatic cancer is needed for better patient management.

In this study, we investigated the correlation between CE-US findings and pathologically measured MVD in pancreatic cancer. We also investigated the clinicopathological significance of CE-US.

\section{MATERIALS AND METHODS}

\section{Subjects}

The subjects were 16 patients who underwent CE-US before surgery and were pathologically diagnosed with pancreatic ductal adenocarcinoma after resection at the Surgery Department of Kurume University between July 2007 and March 2010. A two-year follow-up period up to March 2012 was set. No preoperative chemotherapy or radiotherapy was performed in any of the patients. There were 8 males and $8 \mathrm{fe}$ males aged $63.9 \pm 12.8$ years (mean $\pm \mathrm{SD}$ ), and the tumor-occupied region was located in the pancreas head
$(\mathrm{Ph})$ and body $(\mathrm{Pb})$ of the pancreas in 13 and 3 patients, respectively. The tumor diameter averaged $26.2 \pm 8.57$ $\mathrm{mm}$ (mean $\pm \mathrm{SD}$ ). It was classified into $2 \mathrm{~cm}$ or smaller (TS1) or larger than $2 \mathrm{~cm}$ (TS2-4) following the TS classification in the General Rules for the Study of Pancreatic Cancer [11], and 5 and 11 cases were included in the TS1 and TS2-4 groups, respectively. The applied surgical procedure was pancreatoduodenectomy (PD) (including pylorus-preserving PD (PPPD) and subtotal stomach-preserving PD (SSPPD)) in 13 patients and distal pancreatectomy (DP) in 3. The histologic type was well-differentiated adenocarcinoma in 10 , moderately differentiated adenocarcinoma in 6 , and poorly differentiated adenocarcinoma in 0 . Eight patients each were positive and negative for lymph node metastasis. Microscopic (R1) and macroscopic (R2) residual tumor resections were performed in 2 and 1 patient, respectively. No liver or periaortic lymph node metastasis was noted before or during surgery in any patient. Adjuvant chemotherapy was performed in 13 patients after surgery (Table 1 ). This study protocol was carried out in accordance with The Code of Ethics of the World Medical Association (Declaration of Helsinki) and our institutional review board. Written informed consent was obtained from all patients before CE-US.

\section{CE-US examination}

US and CE-US were performed after 6-hour fasting. CE-US was performed after observation by B-mode US. The devices and conditions used were: imaging system, TOSHIB A Aplio 80 and XG (Toshiba Medical System Corp., Otawara, Japan); sounding probe, PUT375BT; acquisition mode, pulse subtraction-low mode; mechanical index, 0.21-0.34; gain, 70-80; and frame rate, 15 fps. After the intravenous bolus injection of Sonazoid suspension $(0.5 \mathrm{ml})$, the focus was set to the lower margin of the tumor. Observation was started in the non-contrast phase immediately after contrast medium injection, and continued through the arterial phase about $15-20 \mathrm{sec}$ after injection, and the venous phase about 30-45 sec after injection.

\section{Imaging analysis}

Based on the digitally stored images, the region of interest (ROI) was placed in the enhanced area in the tumor, and the luminance $(\mathrm{dB})$ was numerically presented using Image $\mathrm{J}$ (a public domain, Java-based image processing program developed at the National Institutes of Health). TIC was prepared, and the increase from the value before contrast imaging to the maximum value (maximum value - value before contrast imag- 
TABLE 1.

Characteristics of 16 patients with pancreatic adenocarcinoma

\begin{tabular}{|c|c|}
\hline$n=16$ & \\
\hline Age & $37-79($ mean $\pm \mathrm{SD}, 63 \pm 12.8)$ \\
\hline \multicolumn{2}{|l|}{ Gender } \\
\hline Male & 8 \\
\hline Female & 8 \\
\hline Tumor size(mm) & $15-50($ mean $\pm S D, 26.2 \pm 8.57)$ \\
\hline \multicolumn{2}{|l|}{ Location } \\
\hline $\mathrm{Ph}$ & 13 \\
\hline $\mathrm{Pb}$ & 3 \\
\hline \multicolumn{2}{|l|}{ Operation method } \\
\hline PD(PPPD,SSPPD) & 13 \\
\hline DP & 3 \\
\hline \multicolumn{2}{|l|}{ Histological type } \\
\hline Well differntiated & 10 \\
\hline Moderately differntiated & 6 \\
\hline \multicolumn{2}{|l|}{ LN metastasis } \\
\hline $\mathrm{N}(+)$ & 8 \\
\hline $\mathrm{N}(-)$ & 8 \\
\hline \multicolumn{2}{|l|}{ Residual tumor } \\
\hline R0 & 13 \\
\hline $\mathrm{R} 1$ & 2 \\
\hline $\mathrm{R} 2$ & 1 \\
\hline \multicolumn{2}{|c|}{ Adjuvant chemotherapy after surgery } \\
\hline Yes & 13 \\
\hline No & 3 \\
\hline
\end{tabular}

$\mathrm{Ph}$ : Pancreatic head, $\mathrm{Pb}$ : Pancreatic body,

PD: Pancreatoduodenectomy, DP: Distal pancreatectomy, R0: No residual tumor, R1: Microscopic residual tumor, R2: Macroscopic residual tumor

ing) was regarded as the SI (Fig. 1). The patients were divided into those with SI lower and higher than the mean SI (hypo and hyper SI groups, respectively).

\section{Pathologic examination}

Resected specimens were observed by the same pathologist with another physician specialized in pathology. The largest cross-sectional surface of the tumor in the resected specimen was immunohistochemically stained using anti-CD34 and $\alpha$-SMA antibodies [5]. Three areas rich in micro blood vessels were identified under low magnification (40x). The magnification was then increased (200x), and double stainingpositive micro blood vessels were counted in each area. The mean count was adopted as the MVD (Fig. 2).

\section{Statistical analysis}

The correlation between SI and MVD was assessed using Pearson's correlation. In addition, to investigate the clinicopathological significance of CE-US, the tumor diameter, histological type, lymph node metastasis, and outcome (disease-free survival (DFS) after surgery) were compared between the hypo and hyper SI groups.

Data are expressed as mean \pm SD. Differences in the tumor size, histologic type, and lymph node metastasis between the hypo and hyper SI groups were tested using the Student's t-test. DFS was analyzed using the Kaplan-Meier method, and differences between the hypo and hyper SI groups were tested using the logrank test. $\mathrm{P}<0.05$ was considered significant. All the analyses were done using JMP7.0 (SAS institute, Cary, NC, USA). 


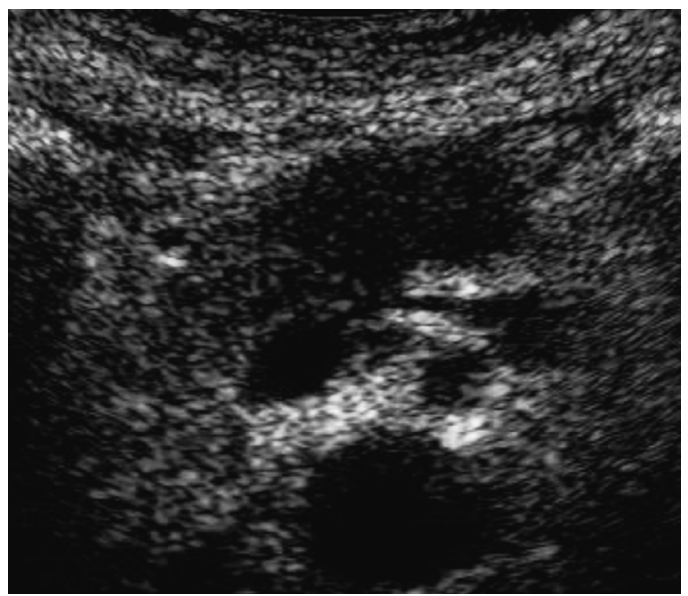

(a)

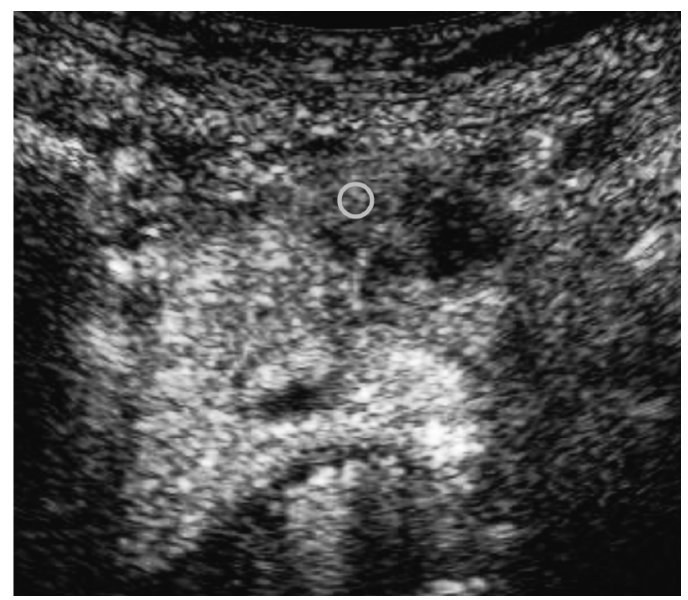

(b)

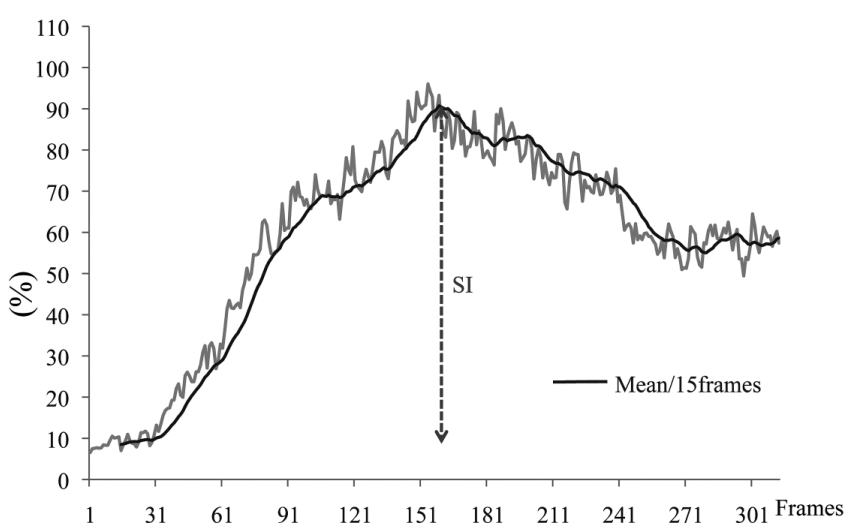

(C)

Fig. 1. (a) Pancreatic adenocarcinoma depicted in the pancreatic body in Pulse Subtraction-low mode US. (b) Pancreatic adenocarcinoma was enhanced by Sonazoid. Circle indicates the ROI. The ROI was placed at the tumor. (c) TIC obtained from the ROI placed at the pancreatic adenocarcinoma. The increment before contrast enhancement to the maximum was regarded as the signal intensity (SI).

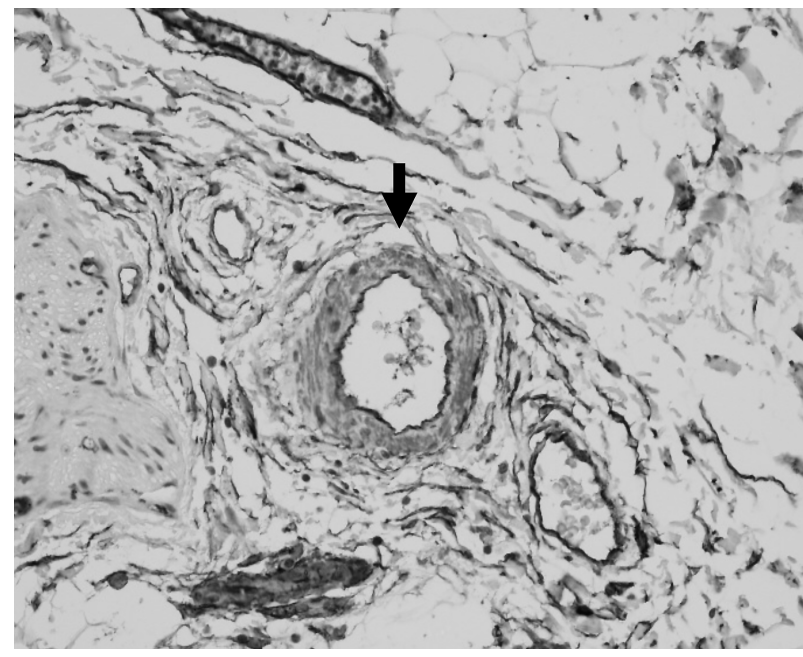

Fig. 2. Immunohistochemical staining of a pancreatic adenocarcinoma specimen with CD34 and $\alpha$-SMA in a $\times 200$ field. Arrow indicates double staining-positive micro blood vessel. Double-stained microvessels were counted, and regarded as the microvessel density (MVD).

\section{RESULTS}

There were no deaths due to surgical complications or other disease in the disease-free survival period. Six patients died of the primary disease during the followup period, and all were included in the hypo SI group. Since no patient died of the primary disease throughout the follow-up period in the hyper SI group, DFS, not overall survival (OS), was selected as an investigation item concerning the prognosis, and it was defined as the period between resection and confirmation of recurrence on follow-up CT. DFS was investigated in all patients and the group with a tumor larger than 2

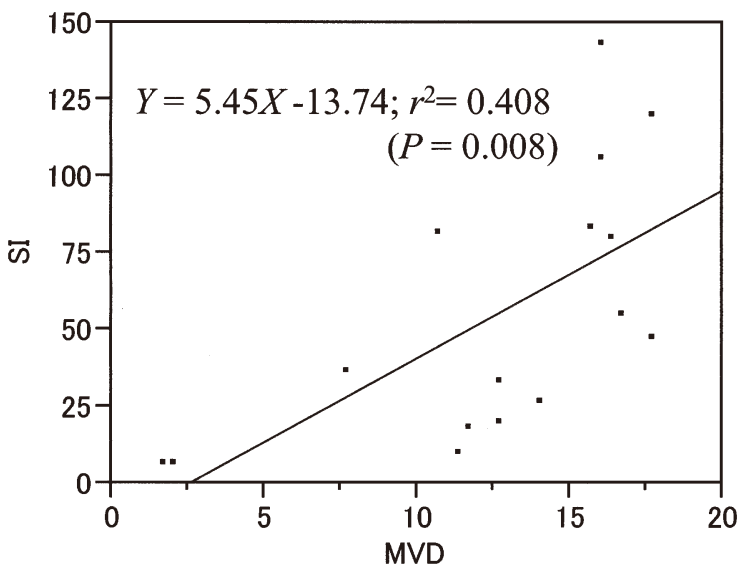

Fig. 3. Pearson's correlation coefficient between SI and MVD. Positive correlations were found between SI and MVD $\left(r^{2}=0.408, P=0.008\right)$. 
$\mathrm{cm}$.

SI in cancerous lesions was 54.6 $\pm 42.9 \mathrm{~dB}, \mathrm{MVD}$ in cancerous lesions was $12.5 \pm 5.02$ arbitrary units, and a correlation $\left(r^{2}=0.408, P=0.008\right)$ was noted between the SI and MVD (Fig. 3).

One $(20 \%)$ of 5 patients with a tumor size smaller than $2 \mathrm{~cm}$ and $8(72.7 \%)$ of 11 patients with a tumor size of $2 \mathrm{~cm}$ or larger were included in the hypo SI group. A significant difference was noted in tumor size between the hypo and hyper SI groups $(\mathrm{P}=0.009)$.

Five $(50 \%)$ of 10 patients with well-differentiated adenocarcinoma and $4(66.7 \%)$ of 6 patients with moderately differentiated adenocarcinoma were included in the hypo SI group. No significant difference was

TABLE 2.

Difference between hypo SI and hyper SI with clinicopathologic parameters.

\begin{tabular}{|c|c|c|c|c|}
\hline & $\mathrm{n}$ & Hypo SI & Hyper SI & $\mathrm{P}$ \\
\hline \multicolumn{5}{|l|}{ Age } \\
\hline$\leq 63$ & 7 & $5(71.4 \%)$ & $2(28.6 \%)$ & 0.280 \\
\hline$>63$ & 9 & $4(44.4 \%)$ & $5(55.6 \%)$ & \\
\hline \multicolumn{5}{|l|}{ Gender } \\
\hline Male & 8 & $5(62.5 \%)$ & $3(37.5 \%)$ & 0.614 \\
\hline Female & 8 & $4(50 \%)$ & $4(50 \%)$ & \\
\hline \multicolumn{5}{|l|}{ Tumor size $(\mathrm{cm})$} \\
\hline$\leq 2$ & 5 & $1(20 \%)$ & $4(80 \%)$ & 0.009 \\
\hline$>2$ & 11 & $8(72.7 \%)$ & $3(27.3 \%)$ & \\
\hline \multicolumn{5}{|l|}{ Histological type } \\
\hline Well-differentiated & 10 & $5(50 \%)$ & $5(50 \%)$ & 0.668 \\
\hline Moderately differentiated & 6 & $4(66.7 \%)$ & $2(33.3 \%)$ & \\
\hline \multicolumn{5}{|l|}{$\mathrm{LN}$ metastasis } \\
\hline $\mathrm{N}(-)$ & 8 & $3(37.5 \%)$ & $5(62.5 \%)$ & 0.151 \\
\hline $\mathrm{N}(+)$ & 8 & $6(75 \%)$ & $2(25 \%)$ & \\
\hline
\end{tabular}

n: Number of patients, Hypo SI: $\leq$ mean SI , Hyper SI: >mean SI, LN: Lymph node, Ph: Pancreatic head, $\mathrm{Pb}$ : Pancreatic body.

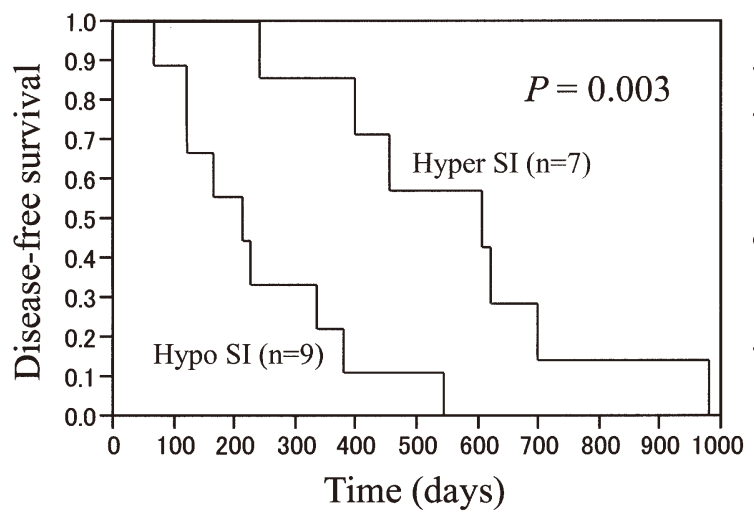

(a)

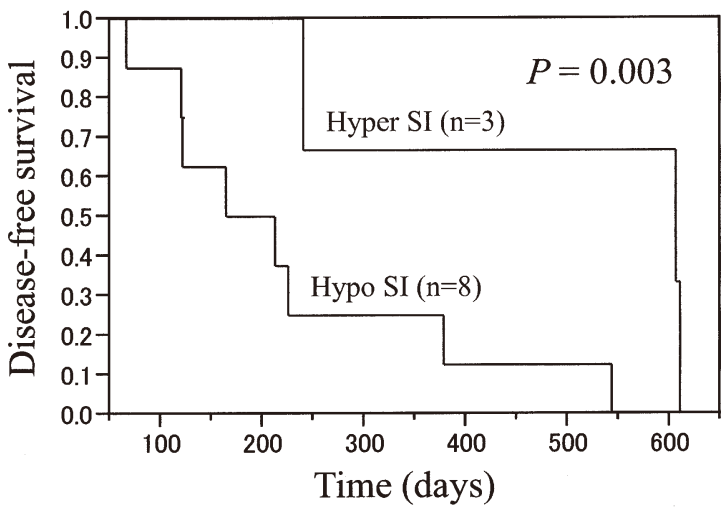

(b)

Fig. 4. (a) Disease-free survival of 16 patients with pancreatic adenocarcinoma according to Hypo SI group and Hyper SI group. The median disease-free survival of the Hypo SI group was 212 days (95\% CI 66, 378). In contrast, the median disease-free survival of the Hyper SI group was 606 days (95\% CI 240, 697). (b) Disease-free survival of 11 patients with pancreatic adenocarcinoma more than $2 \mathrm{~cm}$ in diameter according to Hypo SI group and Hyper SI group. The median disease-free survival of the Hypo SI group was 188 days $(95 \%$ CI 66, 378). In contrast, the median disease-free survival of the Hyper SI group was 606 days $(95 \%$ CI 240,610$)$. 
noted in the histologic type between the hypo and hyper SI groups $(\mathrm{P}=0.668)$.

Three $(37.5 \%)$ of 8 lymph node metastasis-negative patients and $6(75 \%)$ of 8 positive patients were included in the hypo SI group. No significant difference was noted in the presence or absence of lymph node metastasis between the hypo and hyper SI groups $(\mathrm{P}=0.151)$ (Table 2).

The median DFS was 212 days (95\% CI: 66-378) in the hypo SI group consisting of 9 patients and 606 days (95\% CI: 240-697) in the hyper SI group consisting of 7 patients. DFS was significantly shorter in the hypo than in hyper SI group $(\mathrm{P}=0.003)$.

In 11 patients with a tumor size larger than $2 \mathrm{~cm}$, the median DFS was 188 days (95\% CI: 66-378) in the hypo SI group and 606 days (95\% CI: 240-610) in the hyper SI group. Even in the 11 patients with a tumor size larger than $2 \mathrm{~cm}$, DFS was significantly shorter in the hypo (8 patients) than in the hyper (3 patients) SI group ( $\mathrm{P}=0.039)$ (Fig. 4).

\section{DISCUSSION}

Since Weidner et al. initially measured MVD using anti-factor VIII antibody for the staining of micro blood vessels in 1991 [9], many studies on the association between the prognosis of hypervascular tumors, such as breast cancer [12], colon cancer [13], gastric cancer [14], and bladder cancer [15], and MVD have been performed.

Sonazoid ${ }^{\circledR}$, approved in January 2007 in Japan, is an intravenous contrast medium comprised of perflubutane (C4F4) micro bubbles as the active ingredient, which are stabilized with hydrogenated egg-yolk phosphatidylcholine sodium. The mean particle size of the micro bubbles is only $2-3 \mu \mathrm{m}$ [16]. Intravenously administered micro bubbles easily pass through capillary blood vessels in the lung and circulate throughout blood vessels in the whole body. Micro bubbles are phagocytosed by Kupffer cells after circulation but are not transferred from blood vessels, through which pure vascular images and circulatory dynamics can be observed [17-19]. Continuous observation of circulatory dynamics on CE-US became possible employing harmonic imaging at a low acoustic pressure, destroying no micro bubbles. CE-US can be performed in an outpatient consultation room and at bedside in wards, and the procedure is very safe, with an incidence of adverse effects of Sonazoid $\AA$ of only $0.5 \%$, (although administration to patients allergic to eggs or egg products is contraindicated as a rule) [2]. Simplicity and minimal problems with allergy to contrast medium and metals, compared with CT and MRI, are also characteristics of CE-US using Sonazoid®.

The association between CE-US and MVD has been reported in many hyper vascular tumors. Nagase et al. reported that CE-US and completely patent blood vessels were correlated [4]. There have been only a few reports on the association of CE-US and MVD in hypovolemic tumors, such as pancreatic cancer. D'Onofrio et al. reported a strong correlation between the contrast effect of CE-US and CD34-positive blood vessels on pathological examination in 42 pancreatic tumor patients [20]. We prepared TIC from the continuous CE-US images of pancreatic cancer and numerically measured SI, through which we could evaluate contrast enhancement of pancreatic cancer in each patient. Since a correlation was noted between the SI and MVD, CE-US may have visualized only blood flow in pancreatic cancer. All 16 patients underwent preoperative contrast CT, but contrast enhancement was poor in the pancreatic phase in all patients and evaluation of blood flow in each patient was difficult, suggesting the usefulness of CE-US using Sonazoid ${ }^{\circledR}$ for evaluation of blood flow in pancreatic cancer.

DFS was significantly shorter in the hypo than hyper SI group in all 16 patients and in 11 patients with a tumor size exceeding $2 \mathrm{~cm}$. Similarly, poor contrast enhancement of pancreatic cancer was accompanied by metastasis on CE-US in many cases [21] and poorer outcomes in cases with poorer contrast enhancement [22] have been reported. Generally, the tissue oxygen level is determined by diffusion from blood vessels, and tissue $150 \mu \mathrm{m}$ or more distant from blood vessels is considered hypoxic. A hypoxic condition is associated with tumor invasiveness, metastasis, resistance to treatment, and survival rate reduction, serving as a predictive factor in many malignant tumors $[23,24]$. In addition, significantly more patients with a tumor smaller than $2 \mathrm{~cm}$ were included in the hyper SI group. In a nationwide survey in Japan, the 5-year survival rate and MST of patients with 2-cm or smaller pancreatic cancer (TS1) were $31.7 \%$ and 27.4 months, respectively, showing an apparently more favorable prognosis than that of patients with pancreatic cancer larger than $2 \mathrm{~cm}$ (TS2-TS4) $[11,25]$. It was assumed that SI was strong on CE-US in pancreatic cancer with a favorable prognosis, such as TS1, because intratumoral blood flow was retained, whereas SI was weak in highly malignant pancreatic cancer with decreased intratumoral micro-blood vessels.

On the other hand, no significant differences were noted in the histologic type or lymph node metastasis between the hypo and hyper SI groups. A correlation 
between the CE-US findings and histologic type of pancreatic cancer has been reported $[22,26]$. Our study did not include poorly differentiated or undifferentiated adenocarcinoma with advanced fibrosis and severe necrosis. The absence of a significant difference between the hypo and hyper SI groups may have been due to the fact that only well- and moderately differentiated adenocarcinomas were included in this study. An association of lymphangiogenesis and the micro lymphatic vessel density with lymph node metastasis has been reported [27-30]. Since Sonazoid ${ }^{\circledR}$ does not transfer from blood vessels, as described above, intratumoral micro lymphatic vessels are not contrast-enhanced and thus not visualized on CE-US. Accordingly, there was no association between CE-US and the micro lymphatic vessel density, and, subsequently, no significant difference was noted in the incidence of lymph node metastasis between the hypo and hyper SI groups.

In conclusion, the vascularity of pancreatic cancer was successfully evaluated on CE-US using Sonazoid ${ }^{\circledR}$. Since DFS was significantly shorter in the hypo than in hyper SI group, we suggested that CE-US is a useful predictor of patient prognosis after surgery for pancreatic cancer. Since the number of patients in our study was small, further study with more patients is necessary to confirm the usefulness of CE-US.

\section{REFERENCES}

1. Fuminori M. Diagnosis of HCC using ultrasonography. Surgical Therapy 2008; 98:118-132.

2. Mori Y, Hayakawa A, Abe K, Tanigawa M, Takahashi M et al. The safety and efficacy of ultrasound contrast media Perflubutane microbubbles in clinical practice. Jpn J Med Ultrasonics 2011; 38:541-548.

3. Ichikawa T, Haradome H, Hachiya J, Nitatori T, Ohtomo K et al. Pancreatic ductal adenocarcinoma: preoperative assessment with helical CT versus dynamic MR imaging. Radiology 1997; 202:655-662.

4. Nagase M, Furuse J, Ishii H, and Yoshino M. Evaluation of contrast enhancement patterns in pancreatic tumors by coded harmonic sonographic imaging with a microbubble contrast agent. J Ultrasound Med 2003; 22:789-795.

5. Kutami R, Nakashima Y, Nakashima O, Shiota K, and Kojiro M. Pathomorphologic study on the mechanism of fatty change in small hepatocellular carcinoma of humans. Journal of Hepatology 2000; 33:282-289.

6. Sofuni A, Iijima H, Moriyasu F, Nakayama M, Nakamura $\mathrm{K}$ et al. Differential diagnosis of pancreatic tumors using ultrasound contrast imaging. J Gastroenterol 2005; 40:518525.

7. Itoh T, Hirooka Y, Itoh A, Hashimoto S, Kawashima $\mathrm{H}$ et al. Usefulness of contrast-enhanced transabdominal ultrasonography in the diagnosis of intraductal papillary muci- nous tumor of the pancreas. Am J Gastroenterol 2005; 100:144-152.

8. Sofuni A, Itoi $\mathrm{T}$, Itokawa $\mathrm{T}$, Kurihara $\mathrm{T}$, Ishii $\mathrm{K}$ et al. Usefulness of contrast-enhanced ultrasonography in determining treatment efficacy and outcome after pancreatic cancer chemotherapy. World J Gastroenterol 2008; 14:71837191.

9. Winder N, Semple J, Welich W, and Folkman J. Tumor angiogenesis and metastasis: correlation in invasive breast carcinoma. N England J Med 1991; 324:1-8.

10. Japan Pancreatic Society. Pancreatic Cancer Registry Report 2007. J JPN Panc Soc 2007; 22:e20-427.

11. Japan Pancreatic Society. General Rules for the Study of Pancreatic Cancer (The 5th Edition) 2002; Kanehara \& Co., Ltd., Tokyo.

12. Kim SW, Park SS, Ahn SJ, Chung KW, Moon WK et al. Identification of angiogenesis in primary breast carcinoma according to the image analysis. Breast Cancer Res Treat 2002; 74:121-129.

13. Takahashi Y, Kitadai Y, Bucana CD, Cleary KR, and Ellis LM. Expression of endothelial growth factor and its receptor, KDR, correlates with vascularity, metastasis, and proliferation of human colon cancer. Cancer Res 1995; 55:39643968.

14. Tanigawa N, Amaya H, Matsumura M, Shimomatsuya T, and Horiuchi T. Extent of tumor vascularization correlation with prognosis and hematogenous metastasis in gastric carcinomas. Cancer Res 1996; 56:2671-2676.

15. Bochner BH, Cote RJ, Weinder N, Groshen S, Chen SC et al. Angiogenesis in bladder cancer: relationship between microvessel density and tumor prognosis. J Natl Cancer Inst 1995; 87:1603-1612.

16. Sontum PC. Acoustic properties of NC 100100 and their relation with the microbubble size distribution. Invest Radiol 1999; 34:268-275.

17. Suzuki S, Iijima H, Moriyasu F, Sasaki S, Yanagisawa K et al. Differential diagnosis of hepatic nodules using delayed parenchymal phase imaging of levovist contrast ultrasound; Comparative study with SPIO-MRI. Hepatol Res 2004; 29-2:122-126.

18. Giorgio A, Ferraioli G, Tarantino L, de Stefano G, Scala V et al. Contrast-enhanced sonographic appearance of hepatocellular carcinoma in patients with cirrhosis; Comparison with contrast-enhanced helical CT appearance. Am J Roentgenol 2004; 183-5:1319-1326.

19. Quaia E, Calliada F, Bertolotto M, Rossi S, Garioni L et al. Characterization of focal liver lesions with contrast-specific US modes and a sulfur hexafluoride-filled microbubble contrast agent : diagnostic performance and confidence. 2004; 232-2:420-430.

20. D’Onofiro M, Malago R, Zamboni G, Vasori S, Falconi M et al. Contrast-enhanced ultrasonography better identifies pancreatic tumor vascularization than helical CT. Pancreatology 2005; 5:398-402.

21. Masaki T, Ohkawa S, Amano A, Ueno M, Miyakawa K et al. Noninvasive assessment of tumor vascularity by contrast-enhanced ultrasonography and the prognosis of patients with nonresectable pancreatic carcinoma. Cancer 2005; 103:1026-1035.

22. D’Onofrio M, Zamboni GA, Malago R, Mantovani W, 
Principe F et al. Resectable pancreatic adenocarcinoma: Is the enhancement pattern at contrast-enhanced ultrasonography a pre-operative prognostic factor? Ultrasound in Med \& Biol 2009; 35:1929-1937.

23. Vaupel P and Hoeckel M. Predictive power of the tumor oxygenation status. Adv Exp Med Biol 1999; 471:533-539.

24. Harris AL. Hypoxia-a key regulatory factor in tumor growth. Nat Rev Cancer 2002; 2:38-47.

25. Egawa S, Takeda K, Motoki F, Fukuyama S, Sunamura M et al. Small pancreatic cancer: From the national pancreatic cancer registry. J JPN Panc Soc 2004; 19:558-566.

26. Nishida M, Koito K, Hirokawa N, Hori M, Satoh T et al. Does contrast-enhanced ultrasound reveal tumor angiogenesis in pancreatic ductal carcinoma? A prospective study. Ultrasound in Med \& Biol 2009; 35:175-185.

27. Beasley NJ, Prevo R, Banerji S, Leek RD, Moore J et al.
Intratumoral lymphangiogenesis and lymph node metastases in head and neck cancer. Cancer Res 2002; 62:13151320.

28. Maula SM, Luukkaa M, Grenman R, Jackson D, Jalkanen $\mathrm{S}$ et al. Intratumoral lymphatics are essential for the metastatic spread and prognosis in squamaous cell carcinoma of the head neck region. Cancer Res 2003; 63:1920-1926.

29. Hall FT, Freeman JL, Asa SL, Jackson DG, Beasley NJ. Intratumoral lymphatics and lymph node metastases in papillary thyroid carcinoma. Arch Otolaryngol Head Neck Surg 2003; 129:716-719.

30. Straume O, Jackson DG, and Akslen LA. Independent prognostic impact of lymphatic vessel density and presence of low grade lymphangiogenesis in cutaneous melanoma. Clin Cancer Res 2003; 9:250-256. 